\title{
Une nouvelle représentation d'art pariétal dans la Grande grotte de Saint-Front (Domme, Dordogne, France)
}

A new cave art figure in Grande grotte of Saint-Front site (Domme, Dordogne, France)

Éric Robert, Stephane Petrognani, Émilie Lesvignes, Virginie Le Fillâtre, Catherine Cretin et Xavier Muth

\section{OpenEdition}

\section{Journals}

Édition électronique

URL : http://journals.openedition.org/paleo/3610

DOI : $10.4000 /$ paleo.3610

ISSN : 2101-0420

Éditeur

SAMRA

Édition imprimée

Date de publication : 30 décembre 2017

Pagination : 257-266

ISSN : 1145-3370

Référence électronique

Éric Robert, Stephane Petrognani, Émilie Lesvignes, Virginie Le Fillâtre, Catherine Cretin et Xavier Muth, « Une nouvelle représentation d'art pariétal dans la Grande grotte de Saint-Front (Domme, Dordogne, France) », PALEO [En ligne], 28 | 2017, mis en ligne le 01 juin 2018, consulté le 07 juillet 2020. URL : http://journals.openedition.org/paleo/3610 ; DOI : https://doi.org/10.4000/paleo.3610

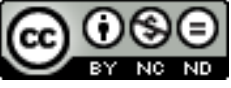

PALEO est mis à disposition selon les termes de la licence Creative Commons Attribution - Pas d'Utilisation Commerciale - Pas de Modification 4.0 International 


\title{
Une nouvelle représentation d'art pariétal dans la Grande grotte de Saint-Front (Domme, Dordogne, France)
}

\author{
Éric ROBERT ${ }^{(a)}$, Stephane PETROGNANI(b), Émilie LESVIGNES ${ }^{(\mathrm{c})}$, \\ Virginie LE FILLÂTRE ${ }^{(d)}$, Catherine CRETIN(e), Xavier MUTH(t)
}

\begin{abstract}
Résumé : Une nouvelle étude a été engagée dans la Grande grotte de Saint-Front depuis 2014, dans le cadre d'un Projet Collectif de Recherche intitulé « Archéologie des sites ornés de Dordogne : cadre conceptuel, potentiels et réalité » (dir. C. Cretin). Considérant les sites ornés comme des témoins archéologiques, ce PCR ainsi que cette étude s'inscrivent dans une dynamique transdisciplinaire visant à associer les témoignages graphiques au matériel archéologique et aux contextes géomorphologique, sédimentologique et paléontologique.

Découverte en 1980, la grotte du Mammouth ou Grande grotte de Saint-Front, près de la commune de Domme, a révélé des représentations sculptées originales et de grandes dimensions, notamment deux mammouths (Aujoulat et al. 1978 ; Delluc et al. 1983). Le reste du dispositif orné s'avère en revanche plus fragmentaire. Dans le cadre des prospections et de la nouvelle analyse des parois ornées, une représentation inédite de tête animale a été mise au jour. Nous présentons ici cette découverte ainsi que la façon dont elle s'inscrit dans une cohérence à la fois technique, par la recherche des volumes, et thématique, pour le secteur de la grotte où elle se trouve.
\end{abstract}

Mots-clés : Mammouth, Saint-Front, grotte, tête animale, volume, art paléolithique.

Abstract: A new cave art figure in Grande grotte of Saint-Front site (Domme, Dordogne, France). A new study was undertaken in the cave of Saint-Front, within the Collectif Research Project entitled « Archéologie des sites ornés de Dordogne: cadre conceptuel, potentiels et réalité» (dir. C. Cretin). Considering decorated sites as archaeological witnesses, this program and this study are part of a transdisciplinary approach aimed at combining graphic evidence with archaeological material and geomorphological,sedimentological and paleontological contexts.

Discovered in 1980, the Mammoth cave or "Grande grotte de Saint-Front", near Domme (Périgord, France), revealed original and massive representations of "bas-relief", including two Mammoth representations (Aujoulat et al. 1978 ; Delluc et al. 1983). The remaining ornate device is more fragmentary. In the prospections and the new analysis framework of the ornate walls, a new representation of animal head was highlighted. We present here this discovery and the way in which it is inscribed in coherence at the same time technical, by the research of the volumes, and thematic for the sector of the cave where it is.

Key-words: Mammoth, Saint-Front, cave, animal head, volume, Palaeolithic art.

(a) MNHN, UMR 7194, équipe Comportements des Néandertaliens et des Hommes anatomiquement modernes replacés dans leur contexte paléoécologique, Paris - eric.robert@mnhn.fr

(b) UMR 7041 ArScAn, équipe Ethnologie préhistorique, Nanterre et CREAP Cartailhac, USR 3414 MSHS Toulouse

(c) CEDARC/Musée du Malgré-Tout, Treignes, Belgique

(d) UMR 5199 PACEA, Université de Bordeaux I Sciences

(e) Musée national de Préhistoire des Eyzies-de-Tayac, UMR 5199 PACEA, Université de Bordeaux I Sciences

(f) Get in Situ 


\section{Introduction}

\section{La Grande grotte de Saint-Front : présentation et problématique}

À un peu plus d'un kilomètre en amont de la ville de Domme, sur la rive gauche de la vallée de la Dordogne, s'ouvrent les grottes de Saint-Front, au-dessus du lieu-dit du même nom (fig. 1). Les falaises coniaciennes qui surplombent ce hameau sont hautes d'une centaine de mètres et abritent de nombreuses cavités, creusées aux dépens d'une série d'accidents tectoniques subparallèles (Aujoulat et Archambeau 1989 ; Le Fillâtre 2016). Plusieurs ont été ornées de gravures ou sculptures : la Grande grotte de Saint-Front ou grotte du Mammouth en fait partie.

L'étude engagée sur la Grande grotte de Saint-Front s'inscrit plus largement dans le cadre d'un Projet Collectif de Recherche intitulé "Archéologie des sites ornés de Dordogne : cadre conceptuel, potentiels et réalité » (dir. C. Cretin), dont l'objectif principal est de veiller à intégrer et croiser l'ensemble des données, graphiques, matérielles et géologiques de ces sites, qui ont parfois été produites séparément (Cretin et al. 2013, 2014).

Ce projet aborde ainsi une sélection des sites ornés entre Dordogne et Vézère (28 grottes) dans une démarche transdisciplinaire commune. Les informations intrinsèques sur ces sites restent souvent limitées, parfois éparses, rendant difficile la définition de leur rôle au sein de l'espace social paléolithique. La démarche engagée dans la grotte de Saint-Front participe de ce travail, et a notamment conduit à associer aux relevés et études du dispositif pariétal, la réalisation de plusieurs sondages dans la cavité destinés à mieux appréhender et mieux intégrer les problématiques archéologiques du sous-sol.

Plus largement, ces recherches se placent dans une dynamique actuelle de relecture des ensembles ornés et de mise en œuvre de nouvelles problématiques d'analyses en grotte ornée.

Depuis 2014, la révision du dispositif pariétal de la Grande grotte de Saint-Front a été engagée, au travers d'une approche associant relevé des tracés pariétaux et des observations géomorphologiques. Elle s'est appuyée notamment sur des outils d'analyse et de numérisation tridimensionnelle pour l'étude des deux mammouths sculptés, de la distribution pariétale et du matériel archéologique au sol.

Avant de présenter la découverte, revenons sur l'historique du site, importante afin d'approcher la diversité des productions et interventions humaines aujourd'hui perceptibles.

\section{La Grande grotte de Saint-Front, historique des découvertes et des travaux d'étude}

La grotte présente la caractéristique d'avoir été fréquentée depuis l'époque paléolithique et occupée à plusieurs reprises jusqu'au $X X^{e}$ siècle. Outre l'époque paléolithique, le matériel archéologique retrouvé dans les sondages de 2014 a permis d'identifier des présences périodes de présence humaine inédites, de l'âge du Bronze final, peut-

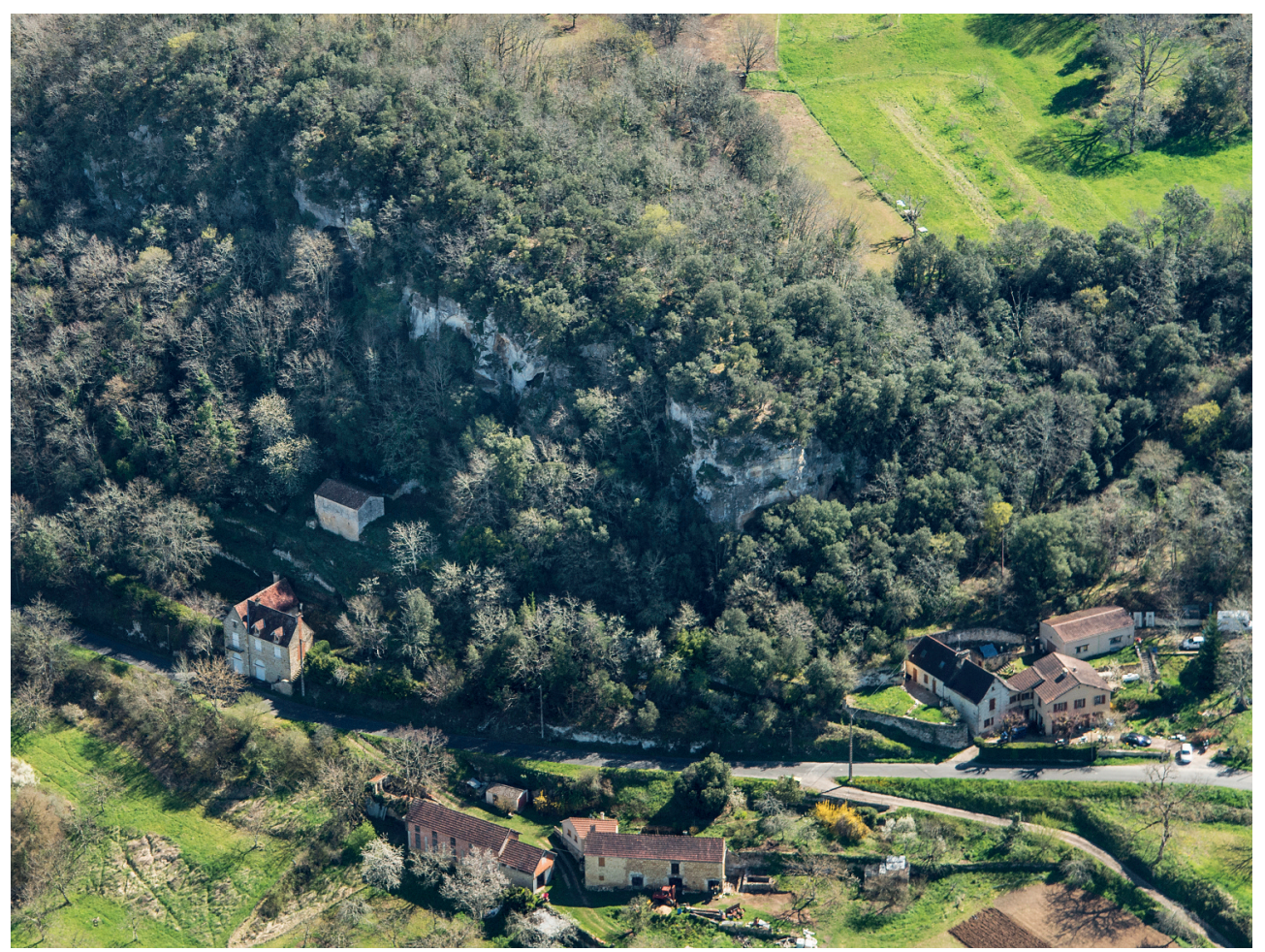

Figure 1 - Vue aérienne de la falaise des grottes de SaintFront. Photo :

É. Lesvignes/CCE sites ornés/PCR Sites ornés, 2015.

Figure 1 - Aerial view of Saint-Front caves cliffs. Photo: É. Lesvignes/CCE sites ornés/PCR Sites ornés, 2015. 
être de l'âge du Fer et des périodes historiques modernes et contemporaines. Certaines de ces occupations successives sont sans doute à relier aux nombreuses traces en paroi, actuellement en cours d'étude'.

La découverte des représentations est liée à celle de la cavité voisine du Pigeonnier (Aujoulat et al. 1978 ; Rigaud 1980 ; Delluc et al. 1983) et s'est faite précisément à la suite d'une visite de la grotte du Pigeonnier à l'initiative de F. Guichard. L'exploration de la Grande grotte de SaintFront a alors permis la découverte du Mammouth sculpté qui a donné son nom à la grotte.

Auparavant, dans le courant du $X X^{e}$ siècle, de nombreuses visites de la cavité eurent lieu, sans que les œuvres ne soient repérées. Cette découverte tardive s'explique certainement par l'état d'altération important du dispositif orné ainsi que la position élevée du mammouth sculpté par rapport au sol actuel (fig. 2), abaissé jadis, suite notamment à l'exploitation de la grotte comme bergerie.

Plusieurs interventions archéologiques ont eu lieu dans différents secteurs de la grotte. Le docteur Bastin aurait procédé à des fouilles, si l'on en croit un courrier du 6 février 1938 adressé à P. Fitte (Delluc et al. 1983, note infrapaginale 11, p. 34), sans toutefois qu'aucune note de fouille ni mobilier archéologique n'aient pour l'instant été retrouvés. Le sol de la grotte est perturbé, certaines excavations se distinguent encore (pied de la paroi gauche, après le second mur d'entrée et dans la partie profonde).

À défaut de niveaux archéologiques en place, un certain nombre d'objets récoltés au cours des nombreuses explorations de la cavité, avant et après les découvertes

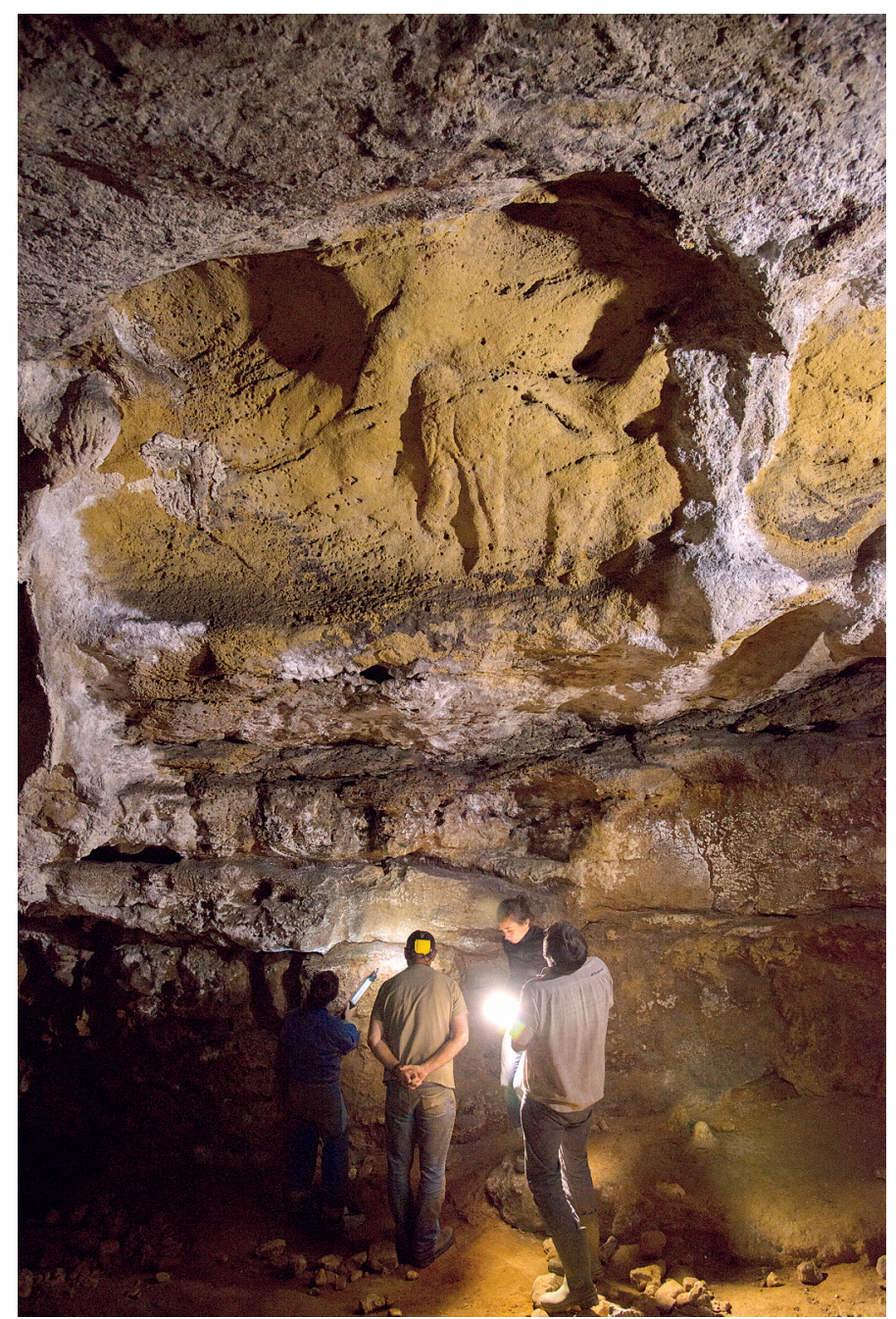

Figure 2 - Mammouth sculpté situé à 4,5 $\mathrm{m}$ du sol actuel. Photo : É. Lesvignes 2013.

Figure 2 - Mammoth carved located at $4.5 \mathrm{~m}$ from the current ground. Photo: É. Lesvignes 2013.

(1) Ce travail, conduit par E. Lesvignes et C. Cretin, porte sur l'ensemble des traces d'activités visibles sur les parois (traces d'outils, isolées ou par zone, traces digitales, noirs de fumée, etc...). Photographiées, inventoriées et classées, elles doivent notamment permettre de mettre en évidence une sériation qui, nous l'espérons, permettra de comprendre leur finalité et leur chronologie relative. 
pariétales, a été mentionné dans plusieurs publications. Des pièces ramassées par des membres du Spéléo-club de Carbon-Blanc (Gironde) et " provenant probablement de la grotte » ont pu être examinées par J.-Ph. Rigaud, qui a identifié des burins de Noailles et des pointes de La Gravette (Rigaud 1980 - p. 405 ; Rigaud 1982 - p. 267). Est également connue une dizaine de pièces lithiques (Delluc et al. 1988 - p. 13-18), dont quatre outils, plusieurs lames et éclats bruts et deux objets en os, collectés par un spéléologue girondin, R. Capdevila, sans qu'aucune attribution chrono-culturelle précise n'ait pu être proposée (Delluc et al. 1988 - p. 13-18). Non documentées, ces pièces n'ont pas encore été retrouvées et n'ont donc pas pu être ré-examinées.

En 2008, suite à la découverte par O. Huard de mobilier archéologique en paroi dans la grotte du Mammouth, M. Peyroux y entreprit une prospection systématique des parois dans le cadre de sa recherche doctorale sur les objets fichés (Peyroux 2012). Elle y a signalé la présence d'un grand nombre de pièces archéologiques (matériel lithique et osseux) sur le sol actuel de la grotte «tant dans la zone antérieure que profonde» (Peyroux, ibid - p. 233), et plusieurs insérées dans les parois (fig. 3). Ces différents constats ont confirmé l'importance du potentiel archéologique du site, au-delà des seules parois, et contribué à lancer une opération de sondages, dirigé par C. Cretin en 2014, qui a permis la collecte d'un important matériel archéologique du Paléolithique supérieur, 572 pièces lithiques (dont 49 outils). Les seules pièces caractéristiques rattachent l'ensemble à un à la charnière entre le Magdalénien moyen et le supérieur (Cretin et al. 2014)

Les parois de la grotte, quant à elles, ont fait l'objet de travaux dans les années 1980 : une analyse des entités graphiques appuyée de relevés et de photographies pour dresser le premier inventaire de la cavité (Delluc et al. 1983), complétée par de nouvelles découvertes (Aujoulat et al. 1989) sur le dispositif pariétal. Au total, 13 entités graphiques ont été répertoriées, avant que nous n'entreprenions notre étude. À ces treize entités s'en ajoute désormais une nouvelle, issue des prospections détaillées que nous avons menées. Tout comme le réexamen de l'ensemble du dispositif pariétal, son identification nous offre un nouveau point de vue sur l'ensemble orné de la grotte du Mammouth (fig. 4).

\section{Formation géologique et structuration spatiale de la cavité}

Bien que voisine de la grotte du Pigeonnier, la Grande grotte de Saint-Front n'appartient pas au même compartiment de faille. En revanche, elle perfore les mêmes formations géologiques du Coniacien moyen ou supérieur, entre un banc massif de calcaire gréseux jaune plurimétrique correspondant au toit de la cavité et des bancs de calcaires gréseux plus clairs à gros débit parallélépipédique associés aux parois. Elle a été creusée à la fois sur joint de stratification et sur une faille par une rivière souterraine entre le Pliocène et le Pléistocène ancien (Le Fillâtre 2016), si l'on se reporte notamment aux altitudes des terrasses de la Dordogne de la carte du Bugue (Karnay et al. 1999 ; Konik 1999), ou au début d'encaissement du réseau de la Garonne (Ambert \& Ambert 1995).

L'âge du début de la karstogenèse et les contextes géologique et géomorphologique empêchent l'existence de toute circulation fluviatile au Pléistocène supérieur dans la grotte du Mammouth. Son histoire ancienne explique aussi

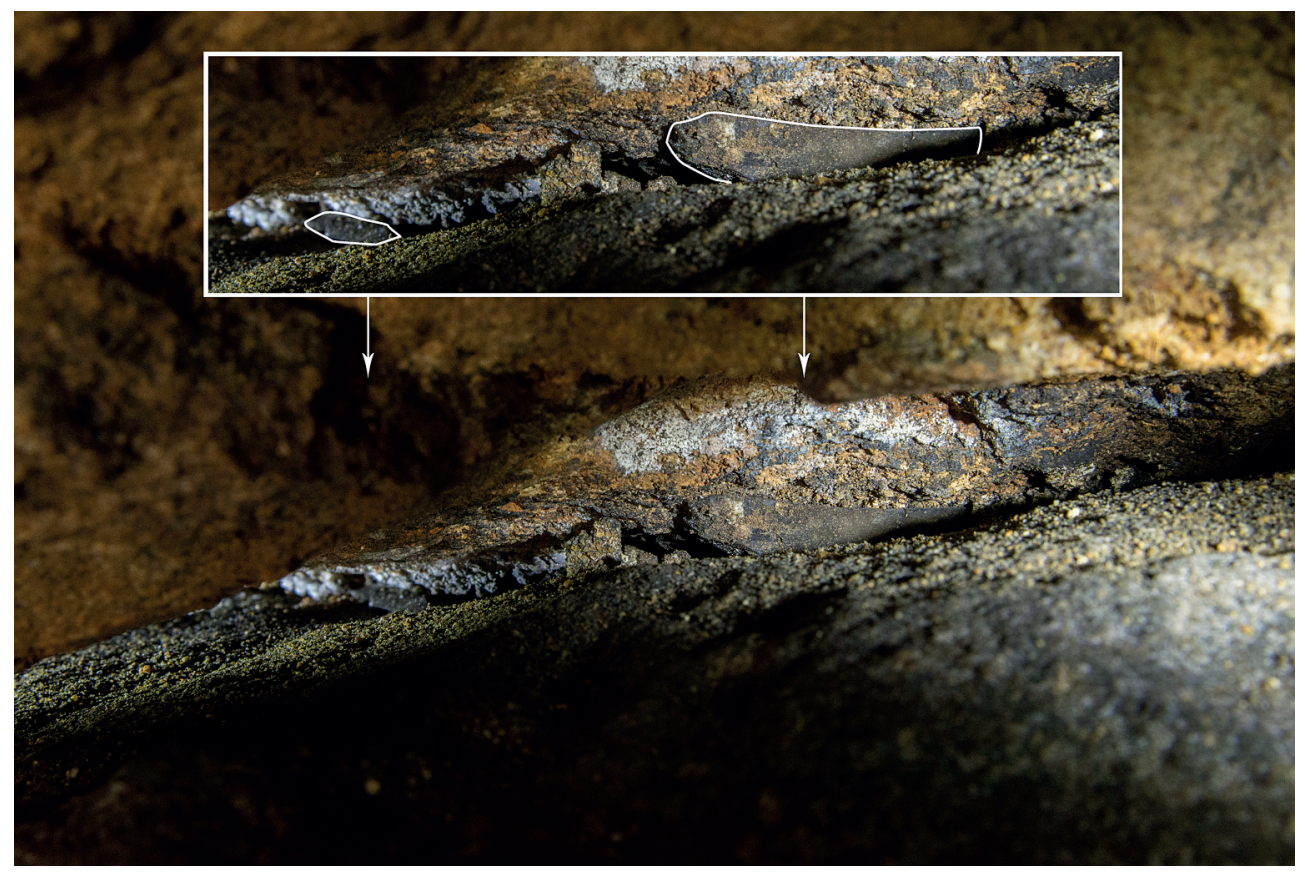

Figure 3 - Objets lithiques fichés dans une fissure, secteur central de la cavité, paroi droite. Photo et DAO : É. Lesvignes 2015.

Figure 3 - Stucked lithic objects in a crack, central sector of the cave, right wall. Photo and CAD: É. Lesvignes 2015. 


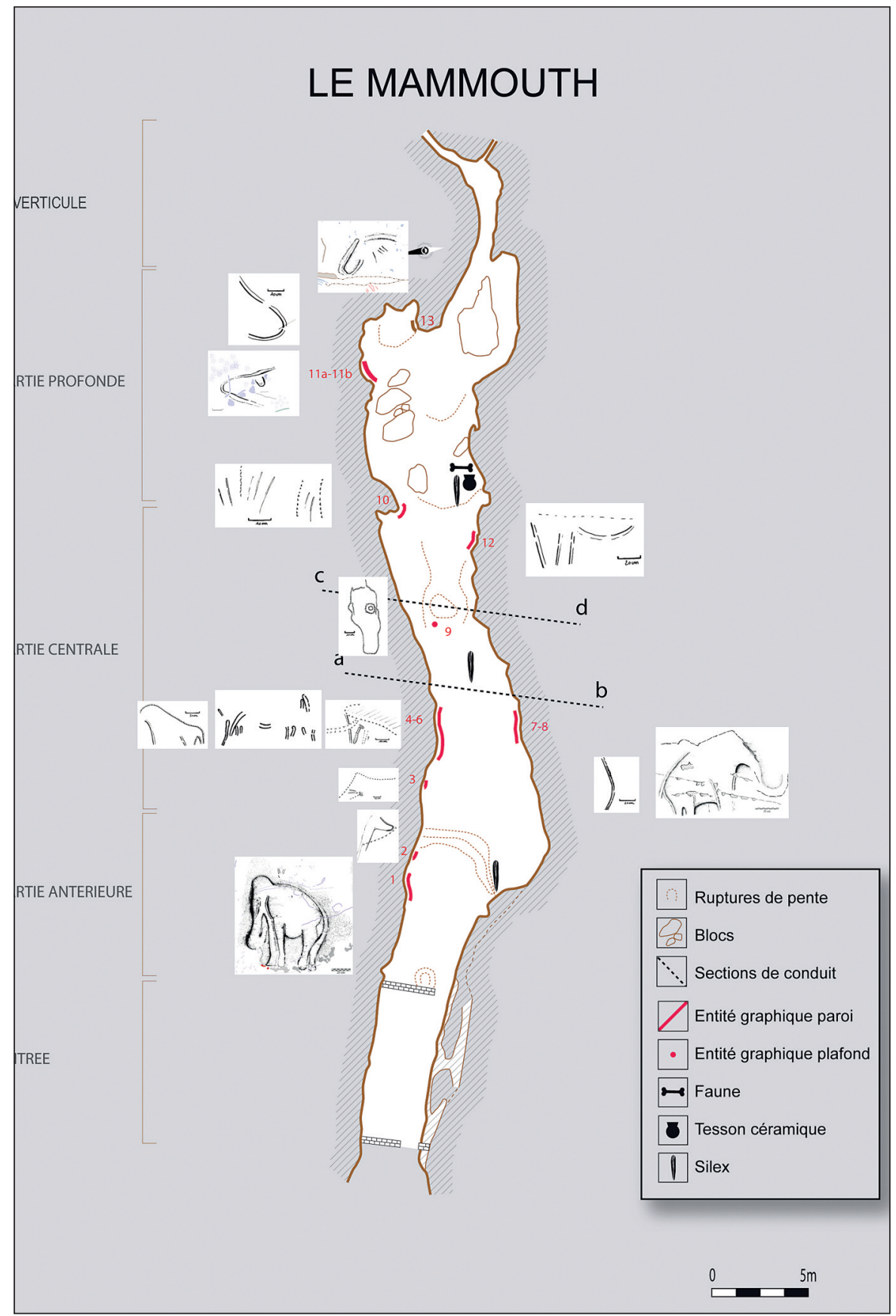

Figure 4 - Plan et sectorisation de la cavité. DAO : N. Cahoreau et

C. Cretin, d'après relevé topographique : B. Galinat 1980, In L'art des cavernes 1984, complété É. Robert.

Figure 4 - Map and sectorisation of the cavity. CAD: N. Cahoreau and

C. Cretin, from topographic survey:

B. Galinat 1980, In : L'art des

cavernes, 1984, completed by É. Robert.

l'état des parois et permet de comprendre et dater une partie des reliefs et microreliefs d'origine naturelle, euxmêmes atténués voire effacés par les altérations chimiques et mécaniques postérieures. Des chronologies relatives ont pu être établies concernant l'évolution de la morphologie du conduit karstique, des formes de paroi, des remplissages et de leurs relations éventuelles avec l'archéologie (art rupestre et occupations humaines) et des fréquentations animales.

Le long circuit karstique de plus de 70 mètres, remontant vers le plateau qui forme la grotte, est subdivisé en quatre zones (fig. 4) : La première, l' « entrée », se situe entre deux murs bâtis. S'ensuit la «partie antérieure » qui constitue la zone d'entrée actuelle où se trouve l'imposant mammouth (entité $n^{\circ} 1$ ). Une pente marque la fin de l'excavation et le début de la «partie centrale». Celle-ci se définit par un sol, plus élevé, une galerie élargie et l'emplacement en vis-à-vis de deux des principaux panneaux de la grotte.

Enfin, la « partie profonde » est séparée de la précédente par un rideau de " pseudo-pendeloques » suspendues à la voûte. Celles-ci sont le produit de l'altération différentielle des grès calcaires par les eaux de la rivière souterraine en 
fonction des proportions de carbonates et de sables de l'encaissant. Des blocs d'effondrements imposants se trouvent en zone profonde, le long de la paroi gauche, et modifient ainsi l'espace de circulation dans ce secteur de la cavité.

L'évolution karstogenétique de la caverne, encore active aujourd'hui, a laissé des traces sur les parois : coupoles, cupules, parois dentelées, cannelures, figures d'écoulement, plaques d'altération, réseau d'entailles liées aux suintements, etc. Associées aux phases d'altération, elles ont pu diminuer certains volumes des représentations et affectent aujourd'hui leur lisibilité.

\section{Présentation du corpus, problématiques de conservation et des états de paroi}

L'inventaire des figurations rassemble deux représentations de mammouth, plusieurs têtes animales, un quadrupède incomplet indéterminé et peut-être les restes d'un second animal (la chute d'une écaille rocheuse aurait emporté les restes de la figure) (fig. 5), ainsi que trois ensembles de traits distincts mais non caractéristiques. À l'exception des deux mammouths, toutes les autres représentations sont incomplètes, et aucune n'offre de détail ou de segment anatomique de nature à proposer des identifications certaines. En outre, elles illustrent bien l'état de conservation médiocre " du fait de la désagrégation du calcaire en résidus sableux dans la zone d'entrée et du recouvrement des surfaces par un voile épais de calcite blanche et sèche dans la zone profonde » (Delluc et al. 1983 - p. 57).

Face à ces difficultés, nous avons donc entrepris un travail spécifique approfondi sur les parois ornées, afin non seulement de procéder au relevé des tracés pariétaux, mais aussi à celui des phénomènes naturels en jeu. L'objectif est de nous assurer du caractère anthropique des entités et tracés étudiés et de comprendre la chronologie des évènements géologiques sur la paroi par rapport aux représentations gravées et sculptées.

Aussi, dans notre travail sur les représentations pariétales, nous avons choisi d'associer au relevé habituel des tracés anthropiques, celui de la géomorphologie pariétale ainsi que des dépôts sédimentaires et chimiques (réalisé par

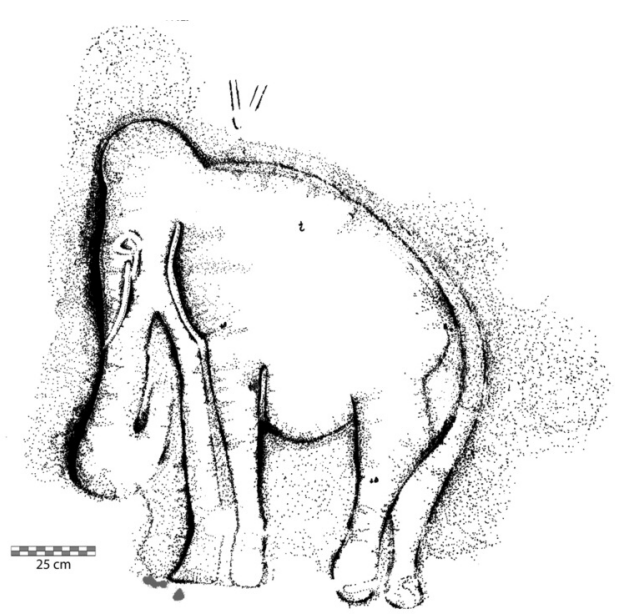

Entité 1

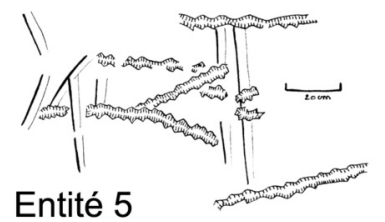

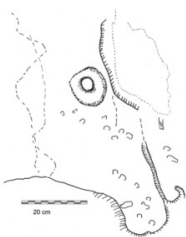

Entité 9

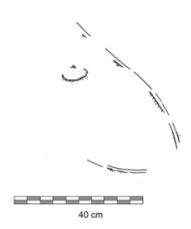

Entité 11b

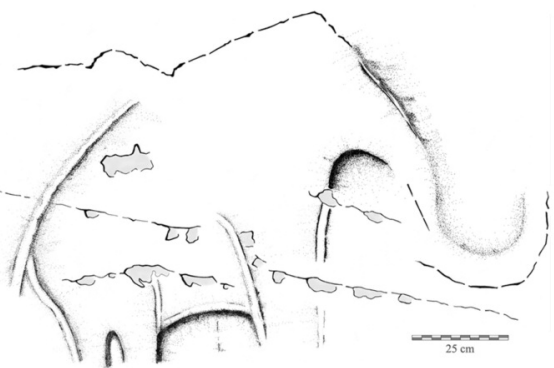

Entité 7
Entité 6

Figure 5 - Planche synthétisant les principales entités graphiques figuratives de la grotte de Saint-Front (doc. S. Petrognani et É. Robert).

Figure 5 - Graphic board of the main figurative entities of $\begin{array}{ll} & \text { the Saint-Front cave (doc. } \\ \text { Entité } 13 & \text { S. Petrognani and É. Robert). }\end{array}$ 
VLF), établi sur un fond photographique commun (réalisé par EL), afin de comprendre la chronologie des altérations, des façonnements naturels des parois et des expressions graphiques anthropiques. Cette méthodologie de relevés combinés constitue une déclinaison originale de l'approche globale de la paroi aujourd'hui en vigueur en art paléolithique et initiée dans les grottes du Lot dès les années 1970 (Lorblanchet et al. 1973). Elle s'inscrit dans une dynamique d'intérêt croissant sur les problématiques liées à la taphonomie des parois (Kervazo et al. 2010 ; Ferrier et al. 2012). Elle a permis une lecture approfondie des parois ornées sur lesquelles la réalisation graphique s'inscrit dans le temps long, dans une histoire reconstituée de la paroi ${ }^{2}$.

\section{Localisation et description de la représentation}

Cette nouvelle entité graphique se situe dans la partie profonde de la cavité ( $n^{\circ} 13$ sur figure 4 ), où les blocs détachés de la paroi limitent l'espace au sol et contraignent la circulation. La morphologie pariétale est aussi différente, notamment par le type d'altérations présentes (fig. 6). Les formations de Mondmilch anciennes et actuelles sur coulées de calcite sont par endroits très abondantes.

L'étude géomorphologique s'est avérée précieuse tant pour la détermination des traits anthropiques anciens que pour la compréhension des volumes.
Au niveau d'une anfractuosité située dans la partie finale de la cavité, face à la paroi nord, plusieurs sillons ont été identifiés. L'un d'entre eux en particulier apparaît profond et vertical, sur un segment de paroi situé juste à droite et en surplomb d'un bloc à ripple-marks (fig. 6). II présente des caractéristiques de volume proches de ce que l'on constate pour le tracé de plusieurs représentations de la cavité.

Le panneau se situe sur un petit banc de grès carbonatés à stratifications obliques, de $25 \mathrm{~cm}$ d'épaisseur, localisé sous les épais grès jaunes massifs de la voûte (fig. 7). Ces grès sont équivalents à ceux sur lesquels a aussi été sculpté le premier mammouth. Un témoin de phases de sédimentations antérieures à l'exploitation de la paroi par les Paléolithiques est observable à proximité du panneau de l'entité 13. II s'agit d'argiles jaunes micacées, de mondmilch, et d'argiles limoneuses brun rouge à fragments de silex et granules et graviers d'hématite. Ces plaquages successifs pourraient d'ailleurs être très anciens et dater de la phase Plio-pléistocène ancien (fig. 8). De plus, sur ce panneau, les traces d'altérations engendrées par les suintements sont très nombreuses. Elles ont dissout localement les carbonates des grès mettant en saillie les grains de quartz, creusant de petites dépressions centimétriques au fond plat et allongé qui rappellent des figures d'écoulement.

L'analyse approfondie de cette paroi altérée a permis de déterminer que le principal sillon vertical était bien profondément gravé, dégageant un volume, et ne s'apparentait à aucun des phénomènes géologiques de la paroi. Son orientation contraste formellement avec les

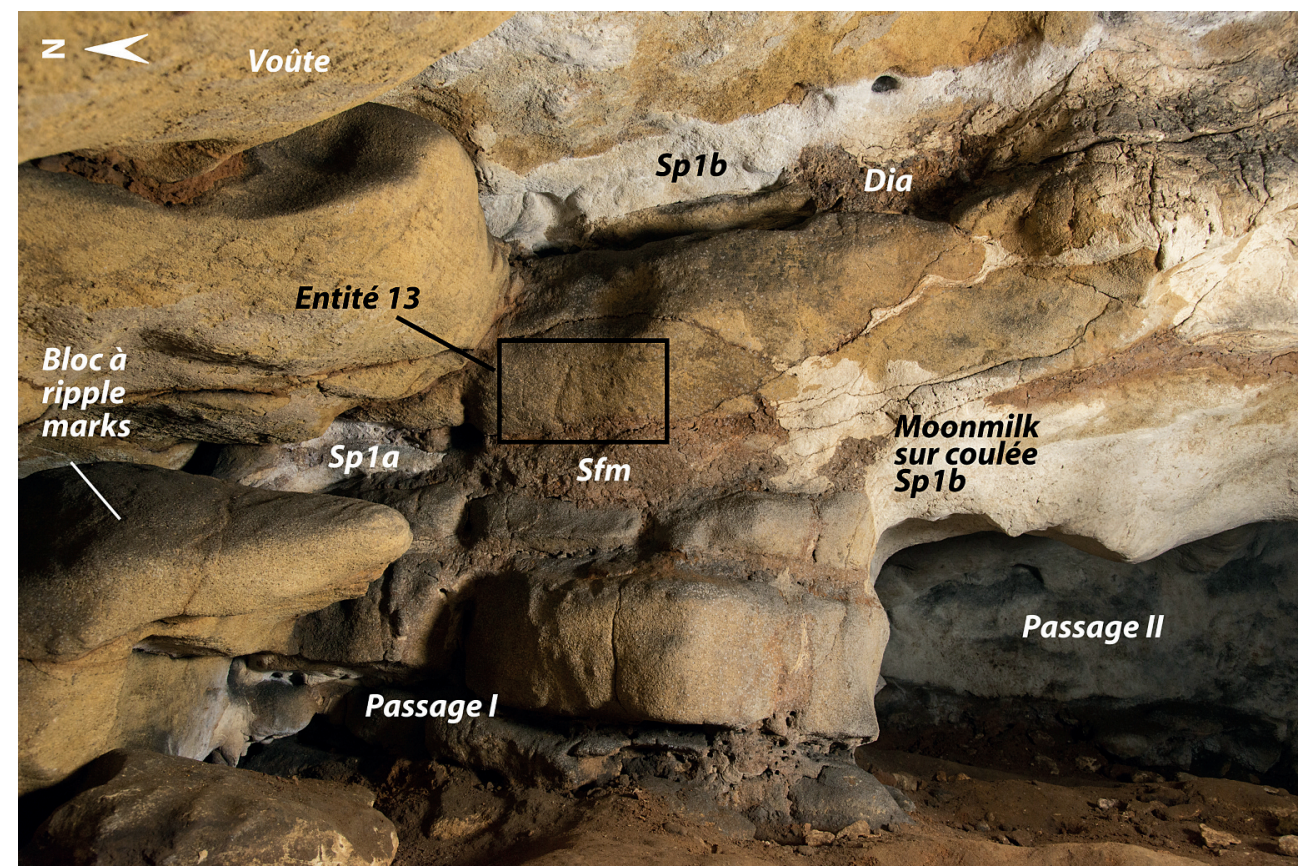

Figure 6 - Situation du panneau de la nouvelle entité $\left(n^{\circ} 13\right)$ dans une anfractuosité du fond de la grotte, au-dessus d'un bloc à ripple-marks. $D A O: V$. Le Fillâtre d'après photo É. Lesvignes 2016.

Figure 6 - Situation of the new entity $\left(n^{\circ} 13\right)$ panel in a recessed area of the bottom of the cave.

CAD: V. Le Fillâtre from photo É. Lesvignes 2016.

(2) Cette méthodologie fait actuellement l'objet d'un article en préparation par l'équipe de recherche de la grotte de Saint-Front. 


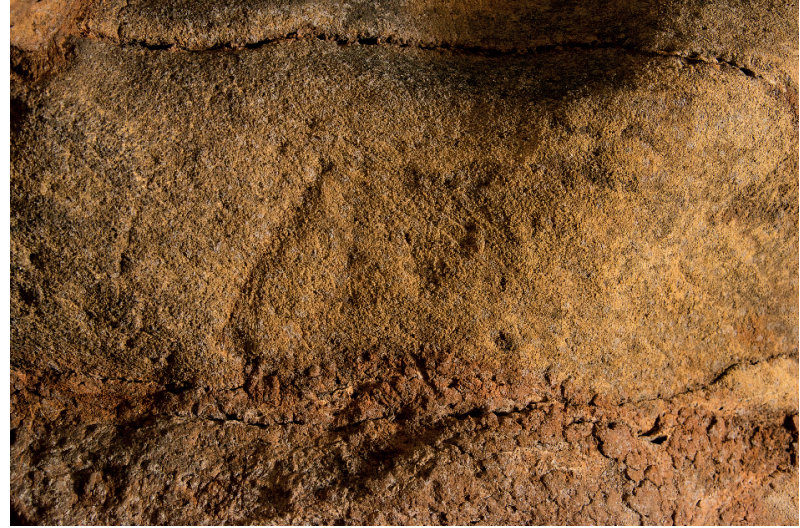

Figure 7 - Panneau de la nouvelle entité graphique ( $\left.n^{\circ} 13\right)$. Photo : É. Lesvignes 2016.

Figure 7 - Panel of the new graphic entity No. 13. Photo: É. Lesvignes 2016.

litages de la stratigraphie et les suintements. Les stratifications de grès calcaires carbonatés apparaissent obliques là où les enlèvements anthropiques principaux sont verticaux ou subverticaux. Le sillon principal ne suit ni les suintements, ni l'orientation de la stratigraphie qu'il recoupe à l'oblique. La courbure gravée à la base de ce sillon, qui produit le relief, traverse plusieurs litages. De plus, le creusement très profond et la largeur du trait sont incompatibles avec la finesse des stratifications obliques. Ces éléments ne peuvent donc être la conséquence de processus géologiques. Ils sont de plus bien distincts des différents types de griffades environnantes (certaines se trouvant sur le panneau), étroites et minces, ainsi que des traces d'enlèvement liés à des outils métalliques, que l'on retrouve à différents endroits de la cavité.

Quelques centimètres à gauche de ces tracés profondément dégagés se trouve un autre sillon vertical qui traverse également les stratifications de la paroi. À la différence du précédent, l'examen approfondi de la paroi a mis en évidence l'absence de stigmate d'origine anthropique. II semble que cette entaille soit le produit de suintements postérieurs au trait, qui ont modifié la surface de la roche.

Cette entité graphique $\mathrm{n}^{\circ} 13$ (fig. 9) couvre un champ total de $26,5 \mathrm{~cm}$ de large pour $18 \mathrm{~cm}$ de haut, situé sur la partie médiane du banc gréseux. Elle se trouve à $140 \mathrm{~cm}$ de hauteur par rapport au sol actuel, probablement proche du

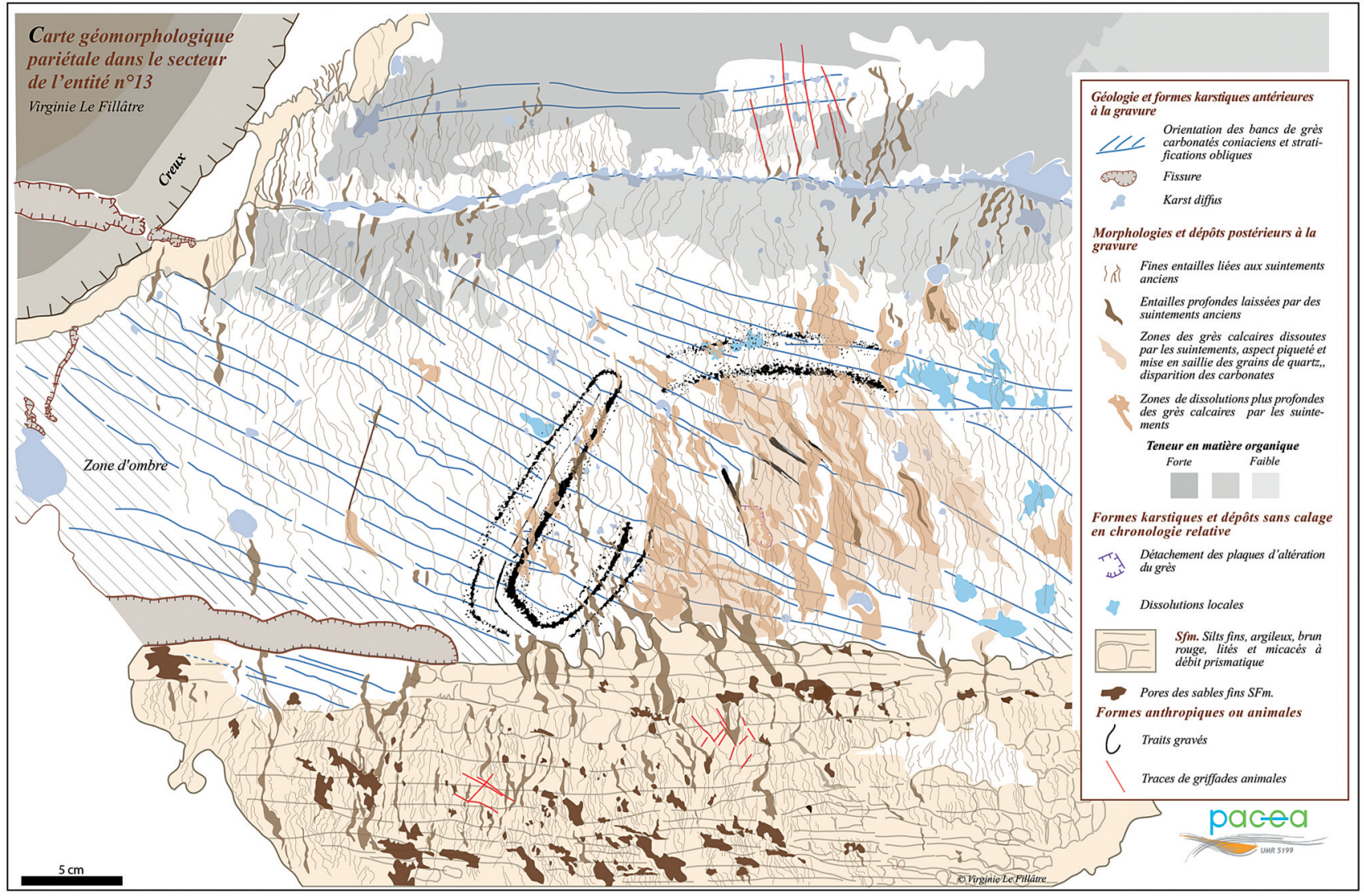

Figure 8 - Cartographie pariétale géomorphologique du panneau de l'entité graphique n`13 (relevé et DAO : V. Le Fillâtre), avec le relevé graphique (É. Robert).

Figure 8 - Geomorphological parietal mapping of the new graphic entity panel (survey and CAD: V. Le Fillatre), with graphic survey layer (survey É. Robert). 


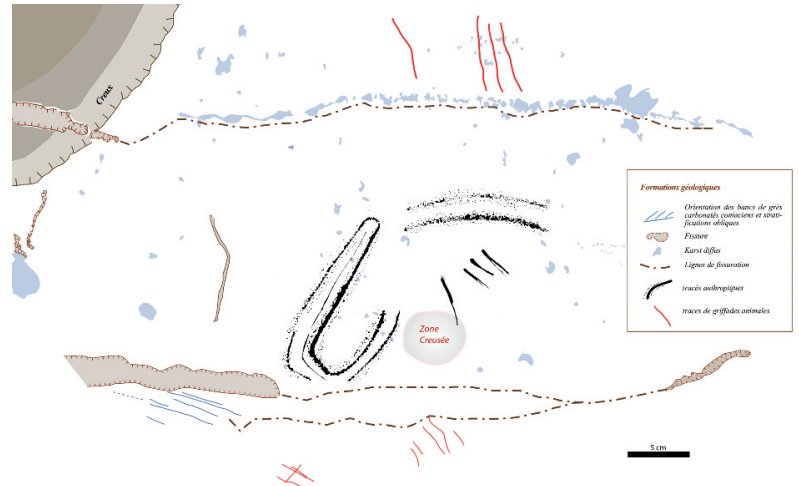

Figure 9 - Relevé (noir et blanc) de la nouvelle représentation $n^{\circ} 13$ (relevé : É. Robert) et éléments géologiques simplifiés antérieurs à la gravure, d'après doc. V. Le Fillâtre (fig. 8).

Figure 9 - Survey of the new representation $N^{\circ} 13$ (survey: É. Robert) and geological elements prior to engraving, according to doc. V. Le Fillâtre (fig. 8).

sol paléolithique. Les nombreuses utilisations de la cavité, ainsi que les fouilles clandestines ne permettent pas de localiser avec certitude le niveau de sol à l'époque paléolithique, bien que des plaquages anciens et des remplissages attribués au Pléistocène supérieur existent encore dans la cavité, notamment dans la zone profonde à une altitude proche des dépôts localisés sous l'entité 13.

Formellement, plutôt qu'une gravure profonde, le sillon principal laisse apparaître une recherche de mise en volume par un creusement à section en $\mathrm{V}$ évasé. La forme et la profondeur de ce tracé ne sont d'ailleurs pas sans rappeler ceux de la tête animale située sur la paroi qui fait presque face à ce panneau, dans le fond de la grotte ${ }^{3}$. Le départ du creusement est apparent au niveau de l'extrémité supérieure du tracé. Cet axe oblique et rectiligne s'incurve en partie inférieure pour remonter ensuite vers la droite de manière moins marquée, mais permet de dégager clairement une tête animale. L'absence de détail anatomique sur le pourtour du tracé, ainsi que celle de l'œil, ne permet pas de proposer une identification spécifique incontestable. Toutefois, l'axe rectiligne extérieur et la relative étroitesse de la courbure inférieure pourraient illustrer le tracé d'un chanfrein et d'un museau arrondi d'un cheval (fig. 10).

Un autre tracé subhorizontal courbe complète cette tête, dans la partie supérieure du panneau. II présente les mêmes caractéristiques techniques de recherche de mise en volume (creusement à section en $\mathrm{V}$ évasé). Bien que le sillon soit moins marqué, il pourrait constituer l'amorce d'une encolure, la crinière dans le cas d'un cheval.

Aucun autre tracé ou dégagement de volume de ce type n'est perceptible dans la continuité de la paroi, qui part ensuite de façon fuyante vers l'axe central de la cavité. À droite de la tête et sous la ligne de la crinière, quatre courts traits gravés sont visibles. Ils ne montrent aucun recoupement avec la figure et présentent un aspect technique distinct (traits étroits et fins), ce qui ne permet pas d'assurer leur contemporanéité. Faut-il les considérer comme un ensemble associé à l'entité graphique (signes ?) ou comme des tracés indépendants ? Au vu de l'absence de détail et de parallèle possible avec d'autres figures de la cavité, ce type d'interprétation reste difficile à établir.

Par ailleurs, plusieurs traces de griffades animales sont visibles à proximité de la nouvelle entité : longues et étroites en haut, plus nombreuses et plus courtes en bas.

Au final, si l'identification d'une tête gravée est assurée par nos observations croisées, il est plus difficile d'assurer la détermination de son espèce. La rectitude du sillon principal associée à sa forte courbure inférieure évoque un chanfrein et un tracé de museau, l'orientation et la légère courbure du second tracé subhorizontal évoquent une encolure. L'ensemble nous conduit à penser qu'il pourrait s'agir d'une tête de cheval, même si le manque de détails anatomiques rend difficile tout jugement définitif. Ce tracé succinct est d'ailleurs cohérent avec d'autres entités graphiques de la cavité. Le large creusement utilisé pour former le contour de la tête rappelle notamment une autre tête animale, l'entité $11 \mathrm{a}$ (fig. 4 et 5) (cf. infra). Ce parallèle formel souligne une cohérence technique et thématique de ce secteur, à savoir les représentations de têtes animales par dégagement de volume.

\section{Une nouvelle représentation en écho à la partie finale du dispositif pariétal ?}

À à peine deux mètres en face de cette nouvelle entité, se trouve une paroi également ornée de plusieurs représentations. La plus remarquable a été identifiée suite à la découverte $\left(n^{\circ} 11\right.$, Delluc et al. 1983) et correspond à une tête animale. L'étude détaillée que nous en avons faite nous a permis de mettre en évidence un contour moins complet que dans la lecture d'origine (fig. 11). C'est surtout la différence d'intensité dans le tracé qui se remarque, sur une surface par ailleurs globalement bien plus homogène et régulière que dans le secteur central. La largeur et la profondeur sont en effet plus importantes sur la partie haute du tracé.

À vingt centimètres à gauche se trouve une autre représentation inventée par Paulette Daubisse en 1984 (entité $11 \mathrm{~b}$ sur figures 4 et 5 ) et mentionnée peu après (Aujoulat et al., 1989). Le tracé est moins profond que pour l'entité 11a. Il semble s'agir d'un contour circulaire évoquant le profil succinct d'une tête animale tournée vers la droite (fig. 12). La lecture est délicate, car le tracé visible apparaît ténu, sur une surface extrêmement concrétionnée. Nos premières observations n'ont permis de mettre en évidence qu'une partie de la lecture initiale, à savoir une ligne rectiligne prolongée par un arrondi (autre possible chanfrein

(3) Il s'agit de l'entité graphique $n^{\circ} 11$ (Delluc et al. 1983), $n^{\circ} 11$ a sur les figures 4 et 5 , relevé figure 11 . 


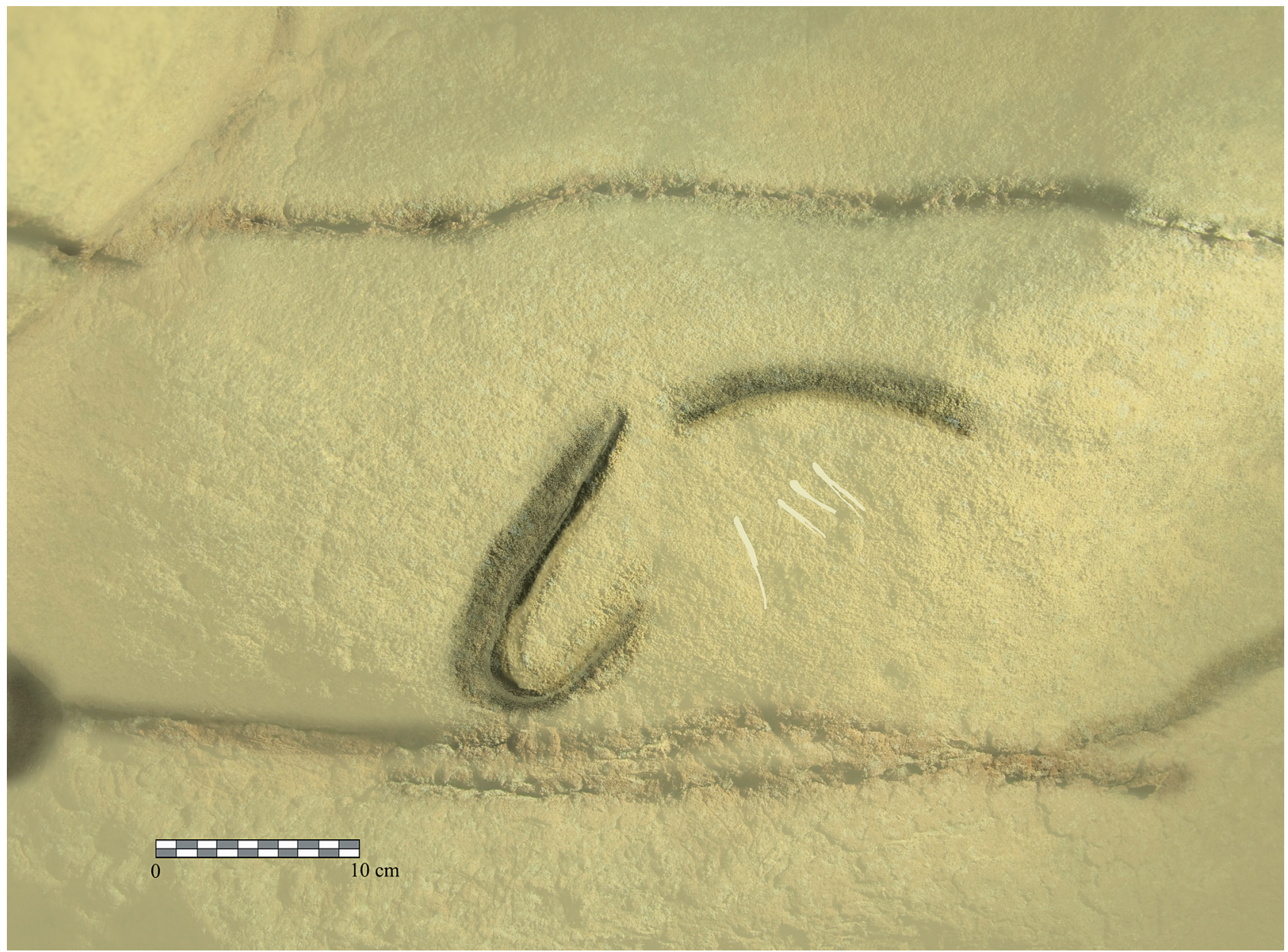

Figure 10 - Relevé infographié de la nouvelle entité n'13 (relevé et DAO : E. Robert) sur fond photographique (E. Lesvignes).

Figure 10 - Infographic survey of the new entity No. 13 (survey and CAD: E. Robert) on a background photographic layer (E. Lesvignes).

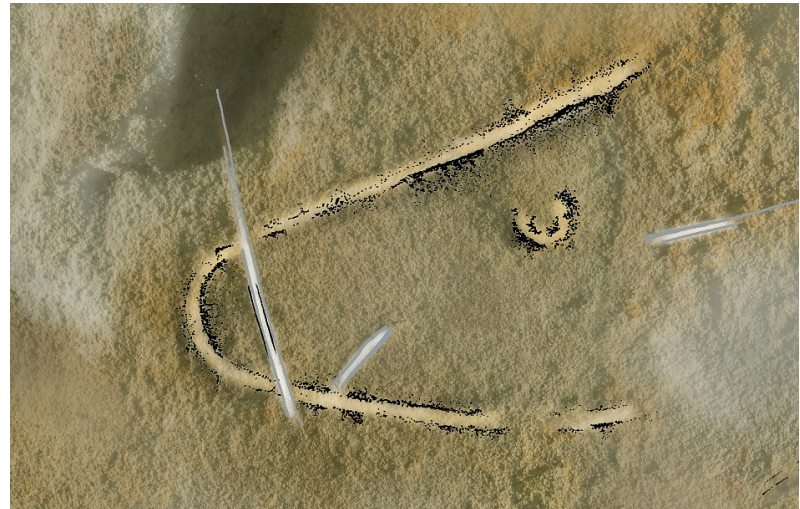

Figure 11 - Relevé infographié de la tête animale $n^{\circ} 11 a$, sur le panneau qui fait face à la nouvelle représentation $n^{\circ} 13$ (relevé et $D A O$ : S. Petrognani, 2016) sur fond photographique (E. Lesvignes 2016).

Figure 11- Infographic survey of the animal head $n^{\circ} 11 a$, on the panel which faces the new representation $n^{\circ} 13$ (survey and CAD: S. Petrognani, 2016) on a background photographic layer (E. Lesvignes 2016). et museau dans la continuité ?). Aucun élément ne nous est apparu pour justifier l'amorce d'une ligne de cou ou de dos. Quant à l'œil, nous nous interrogeons sur sa place et sa nature : seule une petite écaille naturelle nous a semblé en position cohérente par rapport aux tracés gravés.

L'étude de cette paroi se poursuit actuellement afin de préciser au mieux la nature et la complexité de ce panneau.

La proximité de plusieurs têtes animales dans cette partie de la grotte est à relever, car elle offre une apparente cohérence formelle et thématique dans cet espace discret. II faut y ajouter l'interprétation proposée par Norbert Aujoulat d'une autre tête animale, d'équidé, dont le contour n'est formé que par des reliefs naturels et dont seul un tracé circulaire gravé profondément (entité 9 , figures 4 et 5 ) dégage un volume interprété comme un œil (Aujoulat et al. 1989, figure 7 p. 62). Située sous les « dents de pierre » qui marquent l'entrée de la partie profonde de la grotte, cette représentation, si on retient l'interprétation proposée, pourrait en quelque sorte " introduire » la thématique des « têtes animales sans détail » de la partie finale du dispositif pariétal. 


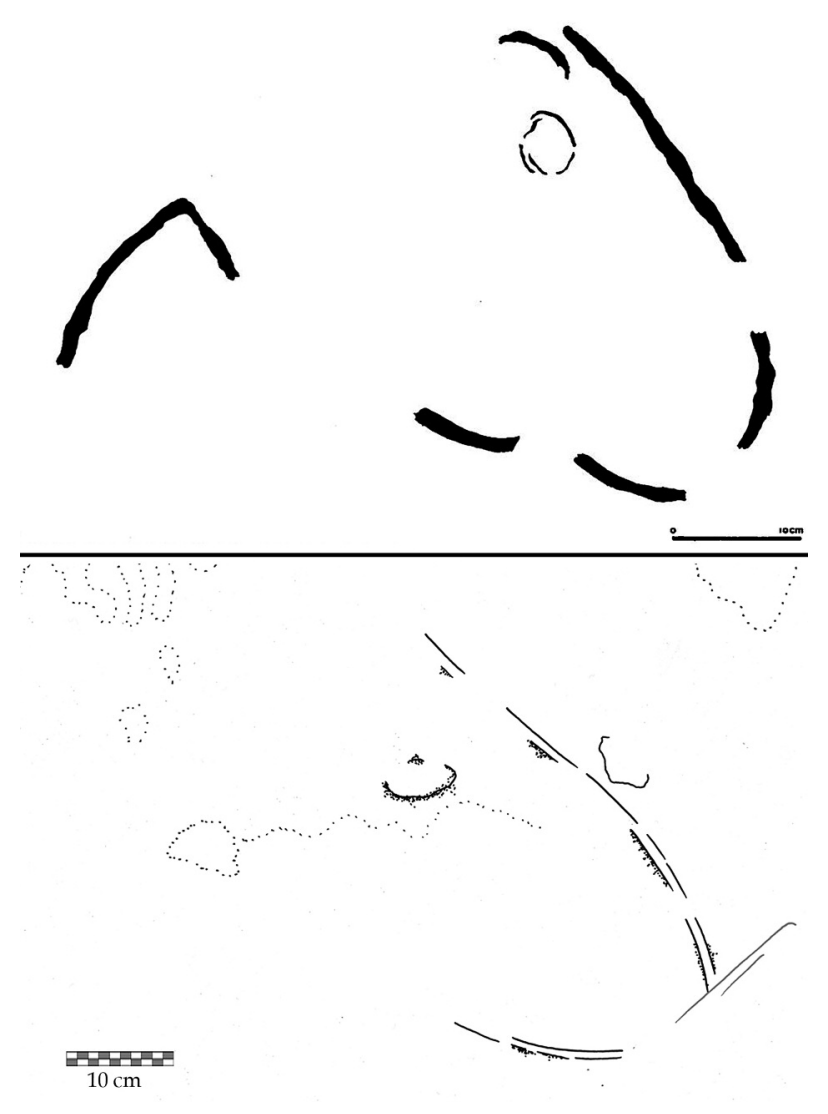

Figure 12 - Entité graphique n०11b. Haut : N. Aujoulat (1989); bas : relevé S. Petrognani 2016.

Figure 12 - Graphical Entity No. 11b. Top: N. Aujoulat (1989); bottom: survey S. Petrognani 2016.

Un dernier aspect mérite d'être souligné concernant la nouvelle entité graphique 13 : le choix de son support. Dans la Grande grotte de Saint-Front, plusieurs des représentations montrent une utilisation des reliefs naturels, soit pour la composition des tracés, soit pour leur cadrage sur la paroi, ce qui illustre une tendance prégnante dans l'art paléolithique (Lejeune 1985 ; Robert 2016). Cette tête animale ne fait pas exception, le museau s'appuyant notamment sur la fissure transversale du banc de grès. De plus, même si le volume a été atténué du fait des processus d'altération, on peut penser que, placée au centre de ce banc de grès, elle offrait une image très marquée, susceptible de faire le pendant des têtes animales situées sur la paroi qui lui fait face, au sein de cette petite alcôve ouverte dans le fond de la cavité. Un emplacement manifestement choisi, sur un support aujourd'hui bien plus induré que la paroi gauche de ce même secteur.

\section{Conclusion : une nouvelle entité graphique à l'image de la grotte du Mammouth}

Si elle ne modifie pas le dispositif pariétal de la cavité, cette nouvelle représentation découverte dans la Grande grotte de Saint-Front illustre en revanche ce qui apparaît comme une thématique dominante dans la partie finale de son dispositif pariétal. Elle témoigne aussi d'une démarche technique privilégiée dans toute la cavité, celle de la construction des images en volume, de la représentation par bas-relief. Cette caractéristique, présente dans la région à travers des abris remarquables tels que Cap-Blanc ou l'abri du Poisson, est ici dominante en grotte. Si l'on peut penser que le mammouth sculpté en demi-relief, près de l'entrée actuelle, pouvait être perceptible avec le passage de la lumière du jour dans la forme originelle de la cavité, il n'en est rien de ces représentations placées au fond de la grotte, dans un espace par ailleurs assez modeste et plutôt discret.

L'intégration chronologique et culturelle du dispositif orné de la Grande grotte de Saint-Front reste encore incertaine, entre périodes anté-magdaléniennes (Delluc et al. 1983 ; Delluc 2014) et Magdalénien (Roussot 1989), mais l'examen croisé des données archéologiques, pariétales et géomorphologiques offre des éléments nouveaux et complémentaires susceptibles d'aider à mieux préciser sa place au sein du paysage préhistorique de la Dordogne. Malgré les différences d'investissement, notamment dans les détails apportés aux représentations (en particulier entre le premier mammouth sculpté et les autres entités), la recherche de la mise en volume, constante dans les différents secteurs de la cavité, plaide pour une homogénéité de l'ensemble.

Cette découverte témoigne enfin de l'importance de réexaminer les parois de grottes au complexe orné modeste, dont les caractéristiques graphiques n'ont pas pu être associées directement à leur contexte matériel, mais qui offrent un potentiel archéologique important. À l'image de recherches récentes comme celles menées à Cussac (Aujoulat et al. 2004, 2013 ; Jaubert et al. 2012), Cap Blanc (Bourdier et al. 2010), dans les grottes des Bernoux (Petrognani et al. 2014 et 2015), de la Mairie (Paillet, ManEstier 2016), du Sorcier (Pigeaud et al. 2012), ou dans les abris Castanet, Blanchard et Cellier (White et al. 2012, 2017 ; Bourrillon et al. 2016), les travaux en cours dans la grotte du Mammouth renouvellent nos connaissances sur les ensembles ornés de Dordogne. Ils permettent aussi d'associer plus étroitement les représentations avec leur contexte archéologique. Ils s'inscrivent en cela pleinement dans la dynamique du PCR dans lequel est conduite cette étude, notamment par la mise en œuvre de lectures croisées associant données graphiques, matérielles, géologiques, et offrent un nouveau point de vue archéologique de ces sites pour mieux appréhender leur contexte.

\section{Remerciements}

Nous tenons tout particulièrement à remercier le propriétaire de la grotte de Saint-Front, M. François Lavergne, qui nous a autorisé à mener ce nouveau programme d'étude dans la grotte du Mammouth et nous a renouvelé son soutien précieux. 
Nos remerciements vont également auprès des équipes du Service Régional d'Archéologie, autour de Nathalie Fourment, conservatrice régionale, ainsi qu'à JeanChristophe Portais, pour leur appui et leur soutien constant à nos opérations de terrain. Ce programme a également bénéficié de l'aide du Conseil départemental de la Dordogne, à qui nous adressons nos remerciements, et tout particulièrement aux équipes du service archéologique dirigé par Mathilde Regeard, qui suivent et appuient nos travaux. Nous tenons aussi à remercier le Centre National de Préhistoire et sa directrice, Geneviève Pinçon, pour ses encouragements et le support apporté à plusieurs de nos travaux. Plusieurs plans et cartes ont pu être réalisés avec la contribution de Nathalie Cahoreau que nous remercions très sincèrement. Nous sommes aussi reconnaissants envers nos laboratoires qui ont apporté aide et appui pour la bonne marche de nos opérations de terrain, le Muséum national d'Histoire naturelle (UMR $7194 \mathrm{HnHp}$ ), I'UMR PACEA à Bordeaux-Pessac, I'UMR ArScAn à Nanterre, le CREAP Cartailhac à Toulouse et le CEDARC en Belgique.

\section{Références bibliographiques}

AMBERT M., AMBERT P. 1995 - Karstification des plateaux et encaissement des vallées au cours du Néogène et du Quaternaire dans les Grands Causses méridionaux. Géologie de la France, n ${ }^{\circ}$, p.37-50.

AUJOULAT N., ARCHAMBEAU C. 1989 - Notes et compléments d'information sur quelques grottes ornées du Périgord Noir. Paleo, $n^{\circ}$ 1, p. 55-64.

AUJOULAT N., DELLUC B. et G., GUICHARD F., PIERRET F. 1978 - Historique des découvertes d'art pariétal à Saint-Front-de-Domme par le Spéléo-Club de Périgueux, Spéléo-Dordogne : Bulletin Spéléo-Club de Périgueux, 67, p. 15-16.

AUJOULAT N., FERUGLIO V., FOURMENT N., HENRYGAMBIER D., JAUBERT J. 2013 - Le sanctuaire gravettien de Cussac (Le Buisson-de-Cadouin, Dordogne, France): premiers résultats d'un projet collectif de recherche. International Newsletter of Rock Art Research INORA, 65, p. 7-19.

AUJOULAT N., GENESTE J.-M., ARCHAMBEAU C., DELLUC M., DUDAY H., HENRY-GAMBIER D. 2004 - La grotte ornée de Cussac - Le Buisson-de-Cadouin (Dordogne) : premières observations. Bull. de la Soc. Préhist. Franç., 99-1, p. 129-137.

BOURDIER C., ABGRALL A., HUARD O., LE BRUN E., PEYROUX M., PINCON G. 2010 - Histoires de bisons et de chevaux : regard sur l'évolution de la frise pariétale de CapBlanc (Marquay, Dordogne) à travers l'analyse du panneau de l'alcôve. PALEO, 21, p. 17-38, 24 fig.

BOURRILLON R., WHITE R., TARTAR E., CHIOTTI L., MENSAN R., CLARK A., CASTEL J.-C., CRETIN C., HIGHAM T., MORALA A., RANLETT S., SISK M., DEVIESE T., COMESKEY D.J. 2016 in press, corrected proof - A new Aurignacian engraving from Abri Blanchard, France: Implications for understanding Aurignacian graphic expression in Western and Central Europe, Quaternary International, http://dx.doi.org/10.1016/j.quaint.2016.09.063

CRETIN C., ARMAND D., BOCHE É., BOURRILLON R., BRUXELLES L., CAMUS H., HOERLE S., KONIK S., LE FILLATRE V., LESVIGNES É., MADELAINE S., MANESTIER E., MORALA A., PETROGNANI S., PLISSON H., ROBERT É., SISK M. 2013 - Archéologie des sites ornés de Dordogne : cadre conceptuel, potentiels et réalité, Programme Collectif de Recherche, Rapport d'opération, Service Régional de l'Archéologie, Aquitaine, 260 p. ill.

CRETIN C., ARMAND D., BOCHE É., BRUXELLES L., CAHOREAU N., CHANCEREL A., DENEUVE E., GENTY D., HCERLE S., KONIK S., LE FILLATRE V., LESVIGNES É., MADELAINE S., MAN-ESTIER E., MANGIER C., MENSAN R., MORALA A., MUTH X., PAILLET P., PETROGNANI S., PLISSON H., ROBERT É., SISK M. 2014 - Archéologie des sites ornés de Dordogne : cadre conceptuel, potentiels et réalité, Programme Collectif de Recherche, Rapport d'opération, Service Régional de l'Archéologie, Aquitaine, 251 p. ill.

DELLUC B. et G. 2014 - Des représentations archaïques de mammouth en Dordogne, Préhistoire du Sud-Ouest, $\mathrm{n}^{\circ} 22,1$ et 2 , p. 41-56.

DELLUC B. et G., avec la collab. E. BAZILE-ROBERT, B. GALINAT, F. GUICHARD et M. OZANNE 1983 - Les grottes ornées de Domme (Dordogne) : La Martine, Le Mammouth et Le Pigeonnier, Gallia Préhistoire, 26, p. 7-80, 52 fig.

DELLUC B. et G., MORALA A., PLISSON H. 1988 Quelques objets des grottes de Saint-Front-de-Domme (Dordogne), Bulletin de la Société Historique et Archéologique du Périgord, 115, Périgueux, p. 11-20 : 7 fig.

FERRIER C., DEBARD E., KERVAZO B., AUJOULAT N., BAFFIER, D., FERUGLIO V., FRITZ C., GELY B., GENESTE J.-M., KONIK S., LACANETTE D., LASTENNET R., MAKSUD F., MALAURENT Ph., PLASSARD F., TOSELLO G. 2012 - Approche taphonomique des parois des grottes ornées. In: L'art préhistorique dans le monde, Actes du Congres IFRAO, Tarascon-sur-Ariège, septembre 2010, Symposium 6 «Datation et taphonomie de l'art pléistocène », J. Clottes dir, p. 192 (livre) et 1071 (CD).

KERVAZO B., FERUGLIO V., BAFFIER D., DEBARD E., FERRIER C., PERROUX A.-S., AUJOULAT N., DELANNOY J.-J., PERRETTE Y. 2010 - Parois et art pariétal : approche taphonomique. L'exemple de la grotte Chauvet (Pont d'Arc). In: Mise en commun des approches en taphonomie, actes du workshop $n 16, X^{*}$ congrès international de l'UISPP, Lisbonne, septembre 2006, p. 4352. Paleo supplément 3.

JAUBERT J., AUJOULAT N., COURTAUD P., DEGUILLOUX M.-F., DELLUC M., DENIS A.,DUDAY H., 
DUTAILLY B., FERRIER C., FERUGLIO V., FOURMENT N., GENESTE J.-M.,GENTY D., GOUTAS N., HENRYGAMBIER D., KERVAZO B., KLARIC L., LASTENNET R.,LEVEQUE F., MALAURENT P., MALLYE J.-B., MORA P., PEMONGE M.-H., PEYRAUBE N.,PEYROUXM., PLISSON $H_{\text {., }}$ PORTAIS J.-C., VALLADAS $H_{\text {., }}$ VERGNIEUX R., VILLOTTE S. 2012 - Le projet collectif de recherche « Grotte de Cussac » (Dordogne, France) : étude d'une cavité ornée à vestiges humains du Gravettien. In: L'art pléistocène dans le monde / Pleistocene art of the world/Arte pleistoceno en el mundo. Actes du Congrès IFRAO, Tarascon-sur-Ariège, septembre 2010e Symposium «Art pléistocène en Europe ", $n^{\circ}$ spécial de Préhistoire, Art et Sociétés, Bulletin de la Société Préhistorique Ariège-Pyrénées, LXV-LXVI, 2010-2011, Livre synthèse, pp. 62-63. CD : 325-342.

KARNAY G., AUJOULAT N., KONIK S., MAUROUX B., PLUCHERY E. TURQ A. 1999 - Notice explicative, Carte géologique, France (1/50 000), feuille Le Bugue (807). Orléans : BRGM, 86 p. Carte géologique par G. Karnay.

KONIK S. 1999 - Les dépôts de pente du Périgord noir : distribution et mise en place ; origine des matériaux et étapes de l'évolution des versants. Thèse de l'Université de Paris I Panthéon-Sorbonne, spécialité Géographie, option Géomorphologie, 473 p. ill.

LE FILLÂTRE V. 2016 - Géomorphologie pariétale de la grotte du “ Mammouth » (St Front, Domme), Rapport d'opération 2016-116, Service Régional de l'Archéologie, Aquitaine, 62 p. $41 \mathrm{fig}$

LEJEUNE M. 1985 - La paroi des grottes, premier « mur » support artistique et document archéologique. Art\&Fact , 2, Le mur dans l'art et l'archéologie, p. 15-24

LORBLANCHET M., DELPECH F., RENAULT P., ANDRIEUX C. 1973 - La grotte de Sainte-Eulalie à Espagnac (Lot). Gallia Préhistoire, 16-1, p. 3-62, 57 fig.

PAILLET P., MAN-ESTIER E. 2016 - Des animaux et quelques signes à la Mairie et à l'abri Mège à Teyjat (Dordogne, France), In : GROENEN M. (dir.) Styles, techniques and graphic expression in rock art, 2787, Actes de la session du $17^{\mathrm{e}}$ congrès mondial de l'UISPP, British Archaeological Reports, p. 88-120, 22 fig.

PETROGNANI S., ROBERT E., BOCHE E., CAILHOL D., LUCAS C., LESVIGNES E. 2014 - Au cœur des premières manifestations graphiques du Paléolithique supérieur : Nouvelles découvertes dans la grotte des Bernoux (Dordogne). Bulletin de la Société Préhistorique Française, tome 111,3 , p. 413-432, 16 fig.
PETROGNANI S., ROBERT E., DJEMA H., CAILHOL D., LUCAS C., LESVIGNES E. 2015 - Confronter contexte archéologique et contexte graphique : l'exemple de la grotte des Bernoux (Dordogne), In : COLLADO GIRALDO H. and GARCIA ARRANZ J. J. (eds.) - Symbols in the Landscape: Rock Art and its Context. Actes du $19^{\circ}$ congrès international de I'IFRAO, Session « Around art: the internal archaeological context of decorated caves », Arkeos 37, Tomar, CD p. 455-481, 25 fig., bibliographie.

PIGEAUD R., BERROUET F., BOUGARD E., PAITIER H., POMMIER V., BONIC P. 2012 - La grotte du Sorcier à Saint-Cirq-du-Bugue (Dordogne, France) : nouvelles lectures. Bilan des campagnes 2010 et 2011. Paleo, 23, p. 223-248, 17 fig.

RIGAUD J.-Ph. 1980 - Grottes du Pigeonnier et du Mammouth, Gallia Préhistoire, Informations archéologiques : circonscription d'Aquitaine, t. 23, fasc. 2, p. 403-405.

RIGAUD J.-Ph. 1982 - Le Paléolithique en Périgord : les données du sud-ouest sarladais et leurs implications, thèse de doctorat d'Etat es sciences, Université de Bordeaux, 493 p., 242 fig., 17 tab, p. 260-263.

ROBERT E. 2016 - Le rôle du support dans la construction des images au sein l'art paléolithique européen, In: GROENEN M. (dir.), Styles, techniques and graphic expression in rock art, 2787, Actes de la session du $17^{\mathrm{e}}$ congrès mondial de l'UISPP, British Archaeological Reports, p. 174-195, 19 fig.

ROUSSOT A. 1989 - La sculpture rupestre magdalénienne en Aquitaine, Bulletin de la SHAP, t. CXVI, p. 45-72, 9 fig., 2 tab.

WHITE R., MENSAN R., BOURRILLON R., CRETIN C., HIGHAM T.F.G., CLARKE A.E., SISK M. L., TARTAR E., GARDERE P., GOLDBERG P., PELEGRIN J., VALLADAS H., TISNERAT-LABORDE N., SANOIT J. (de), CHAMBELLAN D., CHIOTTI L. 2012 - Context and dating of Aurignacian vulvar representations from Abri Castanet, France. Proceedings of the National Academy of Sciences (USA), vol. 109, n²2, p. 8450-8455

WHITE R., BOURRILLON R., MENSAN R., CLARKE A.E., CHIOTTI L., HIGHAM T.F.G., RANLETT S., TARTAR E., SISK M. L., MORALA A., SOULIER M.-C. 2017 - Newly discovered Aurignacian engraved blocks from Abri Cellier: History, context and dating. Quaternary international, in press corrected proof, https://doi.org/10.1016/j.quaint.2017.02.001 
